\title{
Understanding Causal Pathways in Cryptococcal Meningitis Immune Reconstitution Inflammatory Syndrome
}

Authors: Joseph N. Jarvis ${ }^{1,2,3,4} *$, Thomas S. Harrison ${ }^{5}$

Affiliations:

1. Department of Clinical Research, Faculty of Infectious and Tropical Diseases, London School of Hygiene and Tropical Medicine, London, UK

2. Botswana Harvard AIDS Institute Partnership, Gaborone, Botswana

3. Botswana University of Pennsylvania Partnership, Gaborone, Botswana

4. Division of Infectious Diseases, Perelman School of Medicine, University of Pennsylvania, Philadelphia PA, USA

5. Centre for Global Health, Institute for Infection and Immunity, St. George's University of London, UK

* Corresponding author: Botswana Harvard AIDS Institute Partnership, Private Bag BO320, Gaborone, BOTSWANA. Email: joseph.jarvis@1shtm.ac.uk

(c) The Author(s) 2018. Published by Oxford University Press for the Infectious Diseases Society of America. All rights reserved. For permissions, e-mail: journals.permissions@oup.com. 
HIV-associated cryptococcal meningitis continues to pose a major clinical challenge. Mortality rates of $20-40 \%$ have been reported in recent clinical trials using currently recommended amphotericinbased treatments[1,2], and the clinical course of illness in those who do survive is frequently complicated by prolonged or recurrent disease[3, 4]. Immune reconstitution inflammatory syndrome (IRIS) occurs in $15-20 \%$ of individuals who survive their initial illness and initiate antiretroviral therapy (ART), causing a further substantial burden of morbidity and mortality[3-6]. Determining the underlying immune pathology of IRIS, with a view to developing effective prevention or treatment and enabling safe and rapid initiation of ART is, therefore, of considerable importance, and a core component of improving patient outcomes from this severe opportunistic infection.

The development of cryptococcal IRIS (C-IRIS) is strongly associated with a poor baseline inflammatory response, an accompanying high organism or antigen load, and rapid immune reconstitution from a low baseline $\mathrm{CD}^{+}$cell count on ART[3, 7-9]. Although the specific host and pathogen attributes leading to the paucity of effective immune responses and defective antigen clearance during the initial cryptococcal meningitis episode are unknown, human studies suggest that persistently elevated cryptococcal antigen levels despite antifungal therapy leads to increased proinflammatory signalling from antigen-presenting cells, with a lack of effective antigen clearance due to the absence of adequate T-cell help[10-14]. Development of cryptococcal IRIS has been strongly associated with high CNS expression of the chemokines MCP-1(CCL2) and MIP-1 $\alpha$ (CCL3) at initial CM presentation and at ART initiation[12, 13] which, following immune restoration with ART, is hypothesized to result in an influx of inflammatory cells into the CNS, excessive dysregulated local inflammation, and IRIS[11-13, 15, 16].

In this issue of the Journal of Infectious Diseases, Yoon et al. report a novel association between plasma antibody responses at the time of ART initiation and the development of C-IRIS in a well characterised patient cohort from South Africa[17]. Lower levels of plasma IgM antibodies to the 
cryptococcal polysaccharide antigen glucuronoxylomannan (GXM), laminarin (Lam) - a $\square-(1.3)$ glucan containing polysaccharide, and pustulan - a $\square$-(1.6)-glucan, and total plasma IgM, were associated with higher risk C-IRIS. Given our lack of detailed understanding of C-IRIS immunopathology, and the focus of most C-IRIS research to date on macrophage / monocyte[8, 16, $18,19]$ and T-cell mediated immunity $[5,12,13,20]$, these findings offer potentially important new insights into the mechanisms of disease and offer interesting avenues for future research. The role of antibody-mediated protection in HIV-related cryptococcal infection remains uncertain[21, 22] and, as Yoon and colleagues discuss in their article, although animal model and in vitro studies[23-27], along with some human data[28-30], support a role for natural antibody immunity in the host defense against cryptococcal infection, the mechanisms involved remain unclear.

The observation that less robust IgM antibody responses to fungal antigens at the time of ART initiation were associated with higher likelihood of subsequent C-IRIS may indicate an important role for antibody-mediated protection during cryptococcal meningitis, and fit with the overarching hypothesis that a poor initial immune response and subsequent failure of effective immune clearance of cryptococcal antigens are key predisposing factors for IRIS. Such attributions of causality must be made with caution, however. The South African patient cohort from which the samples were derived is one of the largest and best characterized to date[3], but remains a relatively small sample. Few data were available regarding baseline characteristics of patients prior to antifungal therapy. And adjustments, which are difficult for multiple factors in a small study, were not made for some of the known predictors of C-IRIS, including CSF cytokine and chemokine levels. Prior studies performing detailed immune-phenotyping in HIV-associated cryptococcal meningitis have demonstrated the complex interplay between innate and adaptive immune response and the close correlation of many of the soluble and cellular immune markers measured[13, 18-20], making interpretation of potential mechanisms of protection problematic. The correlation seen in the current study between IgM levels and $\mathrm{CD}^{+}$cell counts, known to play a critical role in C-IRIS pathogenesis[15], highlights the 
difficulty of untangling the relative contributions of interconnected elements of the effective host immune response, even given the adjustment made for CD4 cell counts in the study.

The findings of Yoon and colleagues provide further impetus for larger prospective studies to better determine the role of the host immune response in HIV-related cryptococcal infection, both to understand causal pathways to C-IRIS and, also, the immune correlates of outcome more generally. These studies should include detailed clinical and microbiological characterization of cases with longitudinal follow-up, sample collection for immune phenotyping, including antibody responses and, ideally, genotyping, with the ultimate aim of identifying pathways amenable to intervention to reduce the high rates of morbidity and mortality due to cryptococcal meningitis worldwide[31]. Pending these studies, efforts should be focused on reducing the incidence of C-IRIS through the use of rapidly fungicidal antifungal drug combinations[1, 2] and appropriately timed ART initiation[32, 33] based on evidence from randomized controlled trials, and ensuring clinicians recognize C-IRIS early and manage cases appropriately. Data from our recent trials suggest that with these steps in place the morbidity related to C-IRIS can be reduced substantially in patients initiating ART following cryptococcal meningitis[1, 4, 34], and C-IRIS should no longer carry the devastating prognosis that has historically been associated with the condition.

Footnotes: Dr. Jarvis reports grants from Gilead Sciences Europe outside of the submitted work. Dr. Harrison reports grants from Gilead Sciences, personal fees from Viamet, Pfizer, and Gilead Sciences outside of the submitted work.

\section{References}

1. Molloy SF, Kanyama C, Heyderman RS, et al. Antifungal Combinations for Treatment of Cryptococcal Meningitis in Africa. N Engl J Med 2018; 378(11): 1004-17.

2. Day JN, Chau TT, Wolbers M, et al. Combination antifungal therapy for cryptococcal meningitis. N Engl J Med 2013; 368(14): 1291-302. 
3. Chang CC, Dorasamy AA, Gosnell BI, et al. Clinical and mycological predictors of cryptococcosis-associated Immune reconstitution inflammatory syndrome (C-IRIS). AIDS 2013;27(13):2089-99.

4. Jarvis JN, Bicanic T, Loyse A, et al. Determinants of mortality in a combined cohort of 501 patients with HIV-associated Cryptococcal meningitis: implications for improving outcomes. Clin Infect Dis 2014; 58(5): 736-45.

5. Boulware DR, Meya DB, Bergemann TL, et al. Clinical features and serum biomarkers in HIV immune reconstitution inflammatory syndrome after cryptococcal meningitis: a prospective cohort study. PLoS Med 2010; 7(12): e1000384.

6. Bicanic T, Meintjes G, Rebe K, et al. Immune Reconstitution Inflammatory Syndrome in HIV-Associated Cryptococcal Meningitis: A Prospective Study. Journal of acquired immune deficiency syndromes (1999) 2009.

7. Longley N, Harrison TS, Jarvis JN. Cryptococcal immune reconstitution inflammatory syndrome. Curr Opin Infect Dis 2013; 26(1): 26-34.

8. Meya DB, Manabe YC, Boulware DR, Janoff EN. The immunopathogenesis of cryptococcal immune reconstitution inflammatory syndrome: understanding a conundrum. Current opinion in infectious diseases 2016; 29(1): 10-22.

9. Chang CC, Lim A, Omarjee S, et al. Cryptococcosis-IRIS is associated with lower cryptococcus-specific IFN- $\gamma$ responses before antiretroviral therapy but not higher T-cell responses during therapy. J Infect Dis 2013; 208(6): 898-906.

10. Wiesner DL, Boulware DR. Cryptococcus-Related Immune Reconstitution Inflammatory Syndrome(IRIS): Pathogenesis and Its Clinical Implications. Curr Fungal Infect Rep 2011; 5(4): $252-61$. 
11. Boulware DR, Bonham SC, Meya DB, et al. Paucity of initial cerebrospinal fluid inflammation in cryptococcal meningitis is associated with subsequent immune reconstitution inflammatory syndrome. The Journal of infectious diseases 2010; 202(6): 962-70.

12. Chang CC, Omarjee S, Lim A, et al. Chemokine Levels and Chemokine Receptor Expression in the Blood and the Cerebrospinal Fluid of HIV-Infected Patients With Cryptococcal Meningitis and Cryptococcosis-Associated Immune Reconstitution Inflammatory Syndrome. J Infect Dis 2013; 208(10): 1604-12.

13. Jarvis JN, Meintjes G, Bicanic T, et al. Cerebrospinal fluid cytokine profiles predict risk of early mortality and immune reconstitution inflammatory syndrome in HIV-associated cryptococcal meningitis. PLoS Pathog 2015; 11(4): e1004754.

14. Akilimali NA, Chang CC, Muema DM, et al. Plasma But Not Cerebrospinal Fluid Interleukin 7 and Interleukin 5 Levels Pre-Antiretroviral Therapy Commencement Predict Cryptococcosis-Associated Immune Reconstitution Inflammatory Syndrome. Clin Infect Dis 2017; 65(9): 1551-9.

15. French MA. Immune reconstitution inflammatory syndrome: immune restoration disease 20 years on. Med J Aust 2012; 196(5): 318-21.

16. Meya DB, Okurut S, Zziwa G, et al. Cellular immune activation in cerebrospinal fluid from ugandans with cryptococcal meningitis and immune reconstitution inflammatory syndrome. The Journal of infectious diseases 2015; 211(10): 1597-606.

17. Yoon HA, Nakouzi A, Chang CC, et al. Association Between Plasma Antibody Responses and Risk for Cryptococcus-associated Immune Reconstitution Inflammatory Syndrome. Journal of Infectious Diseases 2018; IN PRESS.

18. Scriven JE, Rhein J, Hullsiek KH, et al. Early ART After Cryptococcal Meningitis Is Associated With Cerebrospinal Fluid Pleocytosis and Macrophage Activation in a Multisite Randomized Trial. The Journal of infectious diseases 2015; 212(5): 769-78. 
19. Scriven JE, Graham LM, Schutz C, et al. The CSF Immune Response in HIV-1-Associated Cryptococcal Meningitis: Macrophage Activation, Correlates of Disease Severity, and Effect of Antiretroviral Therapy. J Acquir Immune Defic Syndr 2017; 75(3): 299-307.

20. Jarvis JN, Casazza JP, Stone HH, et al. The phenotype of the Cryptococcus-specific CD4+ memory T-cell response is associated with disease severity and outcome in HIV-associated cryptococcal meningitis. J Infect Dis 2013; 207(12): 1817-28.

21. Rohatgi S, Pirofski LA. Host immunity to Cryptococcus neoformans. Future Microbiol 2015; 10(4): 565-81.

22. Mukaremera L, Nielsen K. Adaptive Immunity to Cryptococcus neoformans Infections. J Fungi (Basel) 2017; 3(4).

23. Rachini A, Pietrella D, Lupo P, et al. An anti-beta-glucan monoclonal antibody inhibits growth and capsule formation of Cryptococcus neoformans in vitro and exerts therapeutic, anticryptococcal activity in vivo. Infection and immunity 2007; 75(11): 5085-94.

24. Subramaniam KS, Datta K, Quintero E, Manix C, Marks MS, Pirofski LA. The absence of serum IgM enhances the susceptibility of mice to pulmonary challenge with Cryptococcus neoformans. J Immunol 2010; 184(10): 5755-67.

25. Rodrigues ML, Travassos LR, Miranda KR, et al. Human antibodies against a purified glucosylceramide from Cryptococcus neoformans inhibit cell budding and fungal growth. Infection and immunity 2000; 68(12): 7049-60.

26. Zhong Z, Pirofski LA. Opsonization of Cryptococcus neoformans by human anticryptococcal glucuronoxylomannan antibodies. Infection and immunity 1996; 64(9): 3446-50.

27. Fleuridor R, Lees A, Pirofski L. A cryptococcal capsular polysaccharide mimotope prolongs the survival of mice with Cryptococcus neoformans infection. J Immunol 2001; 166(2): 108796. 
28. Subramaniam K, Metzger B, Hanau LH, et al. $\operatorname{IgM}(+)$ memory B cell expression predicts HIV-associated cryptococcosis status. The Journal of infectious diseases 2009; 200(2): 24451.

29. Rohatgi S, Gohil S, Kuniholm MH, et al. Fc gamma receptor 3A polymorphism and risk for HIV-associated cryptococcal disease. MBio 2013; 4(5): e00573-13.

30. Fleuridor R, Lyles RH, Pirofski L. Quantitative and qualitative differences in the serum antibody profiles of human immunodeficiency virus-infected persons with and without Cryptococcus neoformans meningitis. The Journal of infectious diseases 1999; 180(5): 152635.

31. Rajasingham R, Smith RM, Park BJ, et al. Global burden of disease of HIV-associated cryptococcal meningitis: an updated analysis. Lancet Infect Dis 2017.

32. Boulware DR, Meya DB, Muzoora C, et al. Timing of antiretroviral therapy after diagnosis of cryptococcal meningitis. N Engl J Med 2014; 370(26): 2487-98.

33. Makadzange AT, Ndhlovu CE, Takarinda K, et al. Early versus delayed initiation of antiretroviral therapy for concurrent HIV infection and cryptococcal meningitis in subsaharan Africa. Clin Infect Dis 2010; 50(11): 1532-8.

34. Jarvis JN, Meintjes G, Rebe K, et al. Adjunctive interferon-gamma immunotherapy for the treatment of HIV-associated cryptococcal meningitis: a randomized controlled trial. AIDS (London, England) 2012; 26(9): 1105-13. 